\title{
TMS Member Receives ABET Honor; Congressional Fellow Update; Welcome New Members
}

\section{Jeffrey Fergus Named ABET Fellow}

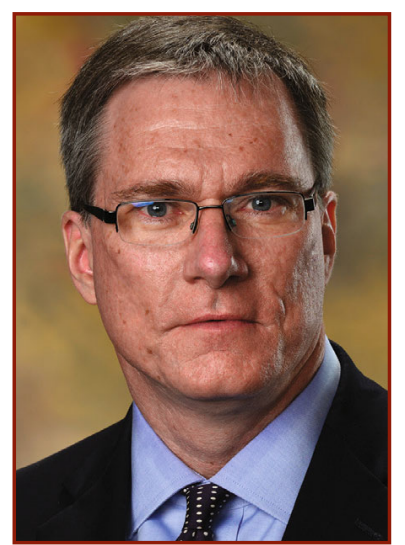

Jeffrey Fergus

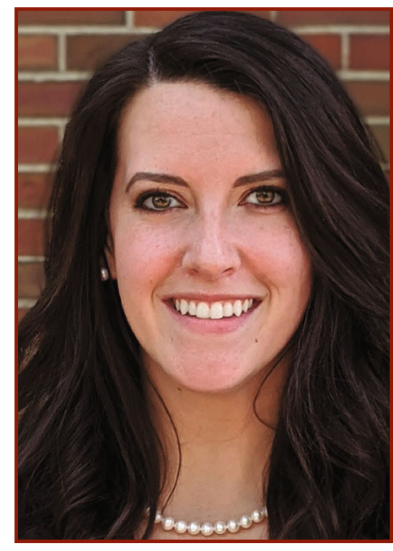

Megan Malara
Jeffrey Fergus, associate dean for undergraduate studies and program assessment and professor of materials engineering in the Samuel Ginn College of Engineering at Auburn University, was chosen as one of four 2020 ABET Fellows. As noted in his citation, Fergus was selected "for leadership ensuring high-quality training of Program Evaluators and Team Chairs, a smooth transition to the new engineering criteria, and laying the foundation for virtual visits within the EAC." Fergus was honored at the virtual 2020 ABET Awards Celebration in October 2020.

Fergus has held a number of roles within ABET since 2003, most recently as Engineering Accreditation
Commission (EAC) Chair. The EAC is one of four ABET commissions responsible for reviewing educational programs and making accreditation decisions for each program. His additional roles within ABET include Program Evaluator, EAC vice chair of operations, and EAC Executive Committee member, for both TMS and ABET.

As a TMS member for more than 30 years, he has been a past chair and member of the Accreditation and Professional Registration Committees, was the first chair of the Professional Development Committee, and was a Board Director for Professional Development.

\section{TMS/MRS Congressional Fellow Accepts Placement}

Megan Malara, 2020-2021 TMS/MRS Congressional Science and Engineering Fellow, is bringing her background in biology, biomaterials, and materials science to the office of U.S. Senator Sherrod Brown (D-OH). Malara began her one-year term as a special legislative assistance on Capitol Hill in September 2020, after a two-week science policy orientation and several rounds of interviews with multiple offices to find the best fit. Activities in this role with Senator Brown's office may include conducting legislative work, assisting in congressional hearings, or preparing briefs and writing speeches.

As a graduate student, Malara attended Material Advantage Congressional Visit Days, where she learned more about the role that scientists can play in advocating for and influencing policy. "Scientists have a duty to the public to inform decisions made by policymakers," she said. She continued her interests as a community team leader for a political campaign where she trained and organized volunteers to engage with the public on policy-related issues. Influenced by her Rust Belt upbringing, Malara has policy interests in translating research to manufacturing jobs, increasing opportunities for education, and improving public confidence in science.

For a listing of past Fellows and their JOM articles highlighting what they learned through the program, visit www.tms.org/PGA and click the link for TMS/MRS Congressional Science and Engineering Fellowship.

\section{member news}

Share the good news about your professional accomplishments! Contact Kaitlin Calva, JOM Magazine Managing Editor, at kcalva@tms.org. Please note that only news submitted by current TMS members will be considered. 


\section{TMS Welcomes New Members}

\section{The TMS Board of Directors approved professional membership for the following individuals at its October 2020 meeting. Please join us in congratulating and welcoming them to all the privileges and benefits of TMS membership.}

\author{
Abbasi, Shahram; Esfahan's \\ Mobarakeh Steel, Islamic \\ Republic of Iran
}

Abdel-Magid, Beckry; Winona State University, United States

Abiodun, Yetunde; University of Lagos, Nigeria, Nigeria

Adekola, Folahan Amod; University of Ilorin, Nigeria

Ahmadvand, Mohammad Saeed; Illinois Institute of Technology, United States

Aidun, Daryush; Clarkson University, United States

Al Halwachi, Hussain A.; Aluminium Bahrain, Bahrain

Albayrak, Onder; Mersin University, Turkey

Albrecht, Robert; University of Silesia, Poland

Ali, Zeeshan; National University of Sciences and Technology, Pakistan

Altin, Serdar; Inonu University, Turkey

Amaechi, Ifeanyichukwu; Institut National de la Recherche Scientifique, Canada

Anderson, Magnus J.; United Kingdom

Ashiri, Rouholah; Islamic Azad University, Islamic Republic of Iran

Assadian, Mahtab; Callaghan Innovation, New Zealand

Aworinde, Abraham K.; Covenant University, Nigeria

Bae, Douglas; Arconic, United States

Bagheri, Ramin; Gam Afarin Wheel Co., Islamic Republic of Iran
Balakrishnan, Marimuthu; Kumarasamy College of Engineering, India

Banerjee, Dilip Kumar; National Institute of Standards and Technology, United States

Barbosa, Isabella Tereza; FERRO Senac University Center, Brazil

Basinger, John A.; United States

Bauman, William J.; Baikowski Malakoff Inc., United States

Bawane, Kaustubh K.; Idaho National Laboratory, United States

Bernard, William J.; Surface Combustion, United States

Bhaumik, Subrajit; SRM Institute of Science \& Technology Kattankulathur, India

Bibhanshu, Nitish; Oak Ridge National Laboratory, United States

Bodnar, Richard L.; SSAB, United States

Borunda, Adan; Advanced Material Research Center, Mexico

Braun, Paul V.; University of Illinois, United States

Bridge, John; University of Washington, United States

Buterbaugh, Chris P.; United States

Cai, Qingan; University of California, Riverside, United States

Chan, Alvaro; Workstrings International, United States

Chen, Shuonan; University of California, Riverside, United States

Cheng, Kai-Yuan; United States

Cheung, Noe; University of Campinas, Brazil

Choudhary, Renu; Ames Laboratory, United States
Christiansen-Salameh, Joyce; Keysight Technologies, United States

Cihova, Martina; ETH Zurich, Switzerland

Clarke, Declan; United Kingdom

Cooke, Kavian O.; University of Bradford, United Kingdom

Cora, Omer N.; Karadeniz Technical University, Turkey

Cubillas Martinez, Fernando; Universidad de Sonora, Mexico

Dada, Oluwaseun John; Signature EcoSystems Technologies, Nigeria

Daoush, Walid M.; Imam Mohammad ibn Saud Islamic University, Saudi Arabia

Das, Kaushik; United States

Das, Piyush K.; Veer Surendra Sai University, India

Das, Satyabrata; Council of Scientific \& Industrial Reserach - Advanced Materials and Processes Research Institute, India

Dash, Khushbu; Indian Institute of Technology Madras, India

De Avila Ribas, Morgana; MacDermid Alpha Electronic Solutions, Japan

Decock, Jeroen; Umicore NV, Belgium

Devanshu, Ambrish; KIET Group of Institutions, India

Dhall, Shivani; D.A.V. College, India

Dhokey, Narendra B.; College of Engineering, India

Dutta Majumdar, Jyotsna; Indian Institute of Technology, India 
Economy, Jessica; Boise State University, United States

Efremenko, Vasily Georgijevich; Priazovskyi State Technical University, Ukraine

El-Hussiny, Naglaa A.; Central Metallurgical Research and Development Institute, Egypt

Emerson, Thomas; United Kingdom

Fagiha, Hamza A.; Illinois Institute of Technology, United States

Fechine, Pierre; Universidade Federal do Ceará, Brazil

Finkelstein, Ryan D.; Wyman Gordan Forgings Inc., United States

Flores, Brandhon; Mexico

Gezicioglu, Yavuz; United States

Ghosh, Suddhodhan; Gautschi Engineering $\mathrm{GmbH}$, Austria

Ghosh, Sumit; University of Oulu, Finland

Giarmas, Vaggelis; Alumil SA, Greece

Gogusetti, Renuka; United States

Golen, Erin; Association for Iron \& Steel, United States

Gomez, Andrea; United States

Gorley, Michael; United States

Greene, Rawley B.; United States

Gruber, Georg; BSH Home Appliances, Germany

Gubicza, Jeno; Eotvos Lorand University, Hungary

Gunasegaram, Dayalan R.; Commonwealth Scientific and Industrial Research Organisation, Australia

Gutierrez-Castaneda, Emmanuel J.; Universidad Autónoma de San Luis Potosi, Mexico

Habib, Khaled; Materials Science and Photo Electronics Lab, Kuwait
Habib, Khaled J.; Materials Science Laboratory, Kuwait

Hadi, Syamsul; State Polytechnic of Malang, Indonesia

Hagisawa, Takehito; The Japan Steel Works, Japan

Hahn, Gail; Boeing Company, United States

Haraldsson, Joakim; Linköping University, Sweden

Harris Odum, Nicole L.; AM/NS Calvert, United States

Hassan, Hala Abd El-Hakim; Ain Shams University, Egypt

Hernandez, Javier; Universidad de Sonora, Mexico

Hernandez-Negrete, Ofelia; Universidad de Sonora, Mexico

Hestad, Samantha; Amptek, United States

Heulens, Jeroen; Umicore, Belgium

Hoeger, Leah; Luvata Appleton LLC, United States

Hug, Eric; CRISMAT Laboratory, France

Hussain, Syed Rizwan; National University of Sciences and Technology, Pakistan

Iglesias, Roberto; University of Oviedo, Spain

Iliukha, Nickolai H.; Kyiv University, Ukraine

Imroze, Fiheon; Indian Institute of Technology Madras, India

Iqbal, Khurram; Institute of Business Management, Pakistan

Ishikawa, Hiroki; Idemitsu Kosan, Japan

Islam, Mohammad; King Saud University, United States

Iyen, Cookey; Federal University Wukari, Nigeria

Jagarlaqudi, Krishna S.; Lam Research, India
Jagatramka, Ritesh; United States

Javad, Sofia; Pakistan

Jeon, Junmo; Aalto University, Finland

Johnson-Glauch, Nicole; California Polytechnic State University San Luis Obispo, United States

Julian, Nicholas H.; University of California, Los Angeles, United States

Jungjohann, Katherine; Sandia National Laboratories, United States

Junjie, Shi; China

Kairy, Prattay Deepta; University of Utah, United States

Kajikawa, Koji; Japan Steel Works M\&E Inc., Japan

Kamble, Deepak; Leibniz Institute for Solid State and Materials Research, Germany

Kar, Amlan; Indian School of Mines, India

Karade, Sukhdeo Rao; Council of Scientific \& Industrial ResearchCentral Building Research Institute, India

Karimi, Roshanak; Foolad Mehr Sahand, Islamic Republic of Iran

Kaushik, Pallava; ArcelorMittal Global R\&D, United States

Kavanlouei, Majid; Urmia University, Islamic Republic of Iran

Kennedy, John Rory; Idaho National Laboratory, United States

Khan, Gobinda Gopal; Tripura University, India

Khan, Kunwar Laiq; KIET, India

Khurram, Yaqoob; National University of Science and Technology, Pakistan

Kirik, Ihsan; Bingol University, Turkey 
Kober, Edward; Los Alamos National Laboratory, United States

Koppad, Praveennath G.; Dayananda Sagar College of Engineering, India

Koskelo, Aaron; Los Alamos National Laboratory, United States

Kostryzhev, Andrii G.; University of Wollongong, Australia

Krishnaswamy, Nagarajan; Commarin Petroleum Inc., Canada

Kuang, Huilin; United States

Kulkarni, Ajit; Indian Institute of Technology Bombay, India

Kumar, Atul; Defence Metallurgical Research Laboratory, India

Kumar, Deepak; Indian Institute of Science, Bengaluru, India

Kumar, Kaushlendra; Defence Metallurgical Research Laboratory, India

Kuo, Dong-Hau; National Taiwan University of Science and Technology, Taiwan

Kurniawan, Denni; Universiti Teknologi Brunei, Brunei Darussalam

Kyung, Shik Oh; POSCO, North Korea

Lanje, Amrut Sadashiv; Dr. Babasaheb Ambedkar College of Arts, Commerce \& Science, India

Lanza, Mark S.; Pacific Northwest National Laboratory, United States

Lavanya, S.; Indira Gandhi Center for Atomic Research, India

Le, Bryan T.; United States

Leopold, Leopold; ETH Zurich, Switzerland

Li, Yang; Chongqing University, China

Liang, Wu; Chongqing University, China
Lillard, Jennifer; University of Akron, United States

Liu, Yu-chen; National Cheng Kung University, Taiwan

Liu, Jiawei; Chongqing University, China

Lliukha, Nickolay G.; Kyiv Politechnical University

Longo, Francisco G.; FL\&A, Dominican Republic

Lu, Matthew Y.; United States

Ma, Chi; United States

Makaya, Advenit; European Space Agency, Japan

Malathi, Madhurai; National Metallurgical Laboratory, India

Mandal, Arup Kumar; Indian Institute of Technology (Banaras Hindu University), Varanasi, India

Manivasagam, Geetha; Vellore Institute of Technology, India

Manzoor, Faisal; University of Ulster, United Kingdom

Marchel, Zeke S.; Oshkosh Corporation, United States

Maria Pillai, Nandhakumaran; Cosma Casting Michigan (Magna International), United States

Martinez, Dora I.; Universidad Autónoma de Nuevo León, Mexico

Matin, M.D.; Bangladesh University of Engineering and Technology, Bangladesh

Meisels, Aryeh P.; Aerojet Rocketdyne, United States

Mhlanga, Cynthia; University of Zimbabwe, Zimbabwe

Mihajlovic, Ivan; University of Belgrade, Serbia and Montenegro

Mir, Feroz; BGSB University, India

Mitchell, Jeremy N.; Los Alamos National Laboratory, United States
Moelnaas, Haavard; Elkem ASA, United States

Mogaddas, Ehsan; Mobarake Steel Co., Islamic Republic of Iran

Momeni, Amir; Hamedan University of Technology, Islamic Republic of Iran

Monsegue, Niven; Federal-Mogul Corporation, United States

Moser, Newell; National Institute of Standards and Technology, United States

Motshwawedi, Saasebeng S.; South Africa

Muhammad, Adeel; Pakistan

Murden, Patrick; United Kingdom

Mustapha, Kabiru; Kwara State University, Nigeria

$\mathrm{Na}$, Noori; United States

Nassar, Eman Ebrahim; Higher Technological Institute, Egypt

Naushin, Nushrat; Khulna University of Engineering and Technology, Bangladesh

Nawaz, Muddasir; Qatar University, Pakistan

Nayyeri, Mohammad Javad; Islamic Azad University, Islamic Republic of Iran

Nyamukapa, Tapiwa G.; University of Zimbabwe, Zimbabwe

Obara, Richard; Emerson Climate Technologies, United States

Obregon Machado, Javier E.; University of Illinois at Chicago, United States

Osoba, Lawrence O.; University of Lagos, Nigeria

Oster, Simon E.; United States

Ouyang, Jun; United States

Owolabi, Oluwasegun B.; National Engineering Design Development Institute, Nigeria 
Oyatogun, Grace Modupe; Obafemi Awolowo University, Nigeria

Panda, Sujata; Rajiv Gandhi Institute for Steel Technology, India

Parasumanna, Ajeet Babu Kumar; The Automotive Research Association of India, India

Park, Woonsup; Aerospace Corporation, United States

Patel, Pranay M.; United States

Pathak, Ashish; Defence Metallurgical Research Laboratory, India

Perez, Antonino; CIMAV, Mexico

Phale, Prasad S.; The Automotive Research Association of India, India

Poddar, Vaishali Sushil; College of Engineering Pune, India

Popuri, Srinivasa R.; University of the West Indies, Barbados

Poursaman, Samaneh; Aluminium Research Centre (Regal), Canada

Pownceby, Mark lan; Commonwealth Scientific and Industrial Research Organisation, Australia

Prakash, Vikas; Washington State University, United States

Prasad, Kartik; Defence Metallurgical Research Laboratory, India

Rachuri, Sharat Chandra; Defence Metallurgical Research Laboratory, India

Rafailidis, Nikolaos; Alumil Group, Greece

Ragavan, Ramakrishnan; India

Rai, Rajesh K.; MNIT, India

Rajendran, Venkatachalam; Dr. N.G.P. Arts and Science College, India

Raji, Sadiq Abiola; Tshwane University of Technology, South Africa
Rameshkumar, Santhosh; DRDO Young Scientist Laboratory, India

Real-Ramirez, Cesar; Universidad Autonoma Metropolitana, Mexico

Reddy, Madhu; Raytheon Vision Systems, United States

Redmond, Paul; Redmond Metallurgical Consulting, United States

Reidy, John P.; Desktop Metal Inc., United States

Reigel, Marissa M.; Savannah River National Laboratory, United States

Reyes-Dominguez, Ivan A.; Universidad Autonoma de San Luis Potosi, Mexico

Rodrigues Jr., Jose Antonio Miguel; Anglo American Brasil, Brazil

Rosales-Marin, Gilberto; Couordinacion Académica Región Altiplano de la Universidad Autónoma de San Luis, Mexico

Roy, Ankit; Lehigh University, United States

Ruckh, Elena; European Space Agency, Netherlands

Ruiz-Sanchez, Angel; Mexico

Ruopp, Peter G.; PGRI, United States

Sabolsky, Edward M.; West Virginia University, United States

Sahay, Satyam S.; John Deere, India

Sahin, Gorkem; ETH, Switzerland

Salehi, Maryam; Iran University of Science and Technology, Islamic Republic of Iran

Samardzic, Veljko; New Jersey Institute of Technology, United States

Saternus, Mariola; Silesian University of Technology, Poland

Satour, Ohsaki; Japan Steel Works M\&E Inc., Japan

Schroeder, Tyler; United States
Selvam, Kannan; Laxmi Institute of Technology, India

Sevik, Huseyin; Mersin University, Turkey

Shalaan, El-sayed; King Abdulaziz University, Saudi Arabia

Shankar, Vani; Indira Gandhi Centre for Atomic Research, India

Sharma, Radheshyam; United States

Sharma, Pankaj; National Institute of Technical Teachers Training and Research, India

Sharma, S.C.; National Assessment and Accreditation Council, India

Sharma, Subhash; Centro de Nanociencias y Nanotecnología de las Universidad Nacional Autónoma de México, Mexico

Sharma, Sumit Kumar; BIT Sindri, India

Sharma, Vinay K.; Nanyan Technological University, Singapore

Shekhar, Prabhanshu; United States

Shelyug, Anna; Institute of Solid State Chemistry, Russia

Shi, Renhai; University of Science and Technology Bejing, China

Shubrajit, Bhaumik; SRM Institute of Science and Technology, India

Shukla, Shivakant; Oak Ridge National Laboratory, United States

Sidorov, Valeriy; Ural State Pedagogical University, Russian Federation

Singh, Garima; ROTOP Pharmaka, India

Singh, Manjri; Council of Scientific \& Industrial Research, India

Singh, Sradhanjali; Council of Scientific \& Industrial Research, India 
Sisodia, Veenu; Central University of Rajasthan, India

Sivaswamy, Giribaskar; Advanced Forming Research Centre, University of Strathclyde, Glasgow, United Kingdom

Sreenarayanan, Sanjay; Lam Research, India

Sridhar, A.; Defence Met Research Laboratory, India

Srivastava, Anurag; Atal Bihari Vajpayee-Indian Institute of Information Technology and Management, India

Su, Shei Sia; United States

Sun, Binhan; Max-Planck Institute, Germany

Sun, Yaokun; United States

Sun, Yani; United States

Szost, Blanka Angelika; University of Cambridge, Germany

Taheri, A. Karimi; Sharif University of Technology, Islamic Republic of Iran

Tamang, Siddharth; Indian Institute of Technology Delhi, India

Tamimi, Saeed; Advanced Forming Research Centre, University of Strathclyde, United Kingdom

Tan, Danny; United States

Tao, Chen; Chongqing University, China

Tattimani, Mahantesh S.; Smt. Kamala \& Sri Venkappa M.
Agadi College of Engineering \& Technology Lakshmeshwar, India

Taunk, Manish; Indus International University, India

Teja, Surya B.; India

Tewari, Sudhir K.; GE Aviation, United States

Tiammar, Arif S.; PT Wanatiara Persada, Indonesia

Trebukhov, Sergey; Institute Metallurgy and Ore Beneficiation JSC, Kazakhstan

Turgut, Güven; Erzurum Technical University, Turkey

Uysal, Alper; Yildiz Technical University, Turkey

Vamsi, K. V.; University of California, Santa Barbara, United States

Veerababu, R.; Defence Metallurgical Research Laboratory, India

Velazquez, Jesus M.; University of California, Davis, United States

Venegas, Diego; Universidad del BíoBío, Chile

Venkatesh, Pavan Hanu; Bharat Heavy Electricals Limited, India

Villarreal, Julian E.; United States

Vishnumolakala, Krishna; United States

Wakeford, Ryan; Terracon, United States

Wang, Jun; Deakin University, Australia

Wang, Tao; Rio Tinto, United States

Welch, Lynn; Kean Miller, United States
Williams, Adrienne D.; ADee Technologies LLC, United States

Williams, Kevin; Advanced Dynamics Corporation Ltd., Canada

Wright, Ashleigh R.; North Carolina State University, United States

Wu, Yipin; Keysight Technologies, United States

Wu, Yiquan; Alfred University, United States

Xhanti, M.; Russia

Yamanoglu, Ridvan; Kocaeli University, Turkey

Yamanouchi, Takuya; Japan Steel Works M\&E Inc., Japan

Yanala, Srinivasa Reddy; Chaitanya Bharathi Institute of Tech, India

Yao, Yao; Schlumberger, United States

Yoshiya, Masato; Osaka University, Japan

Youssif, Khalid; Aluminum Company of Egypt, Egypt

Zahoor, Mudasar; King Fahd University, Saudi Arabia

Zeka, Bastri

Zhang, Liangzhe; Stress Engineering Service Inc., United States

Zhang, Lin; University of Science \& Technology Beijing, China

Zhang, Shuye; Harbin Institute of Technology, China 